\title{
A174 NICOTINIC ACETYLCHOLINE RECEPTOR ACTIVATION COMPLETELY BLOCKS OSTEOCLASTOGENESIS BY INTERFERING WITH OSTEOCLAST PRECURSOR DIFFERENTIATION IN VITRO
}

Peter Mandl, ${ }^{1,2}$ Silvia Hayer, ${ }^{1}$ Despoina Sykoutri, ${ }^{1}$ Gyula Poór, ${ }^{2}$ Josef S Smolen, ${ }^{1}$ Kurt Redlich ${ }^{1}$ Department of Internal Medicine III, Division of Rheumatology, Medical University of Vienna, Vienna, Austria; ${ }^{2}$ National Institute of Rheumatology and Physiotherapy, Budapest, Hungary

10.1136/ard.2010.149013.17

Background and objective In the last few years, the discovery of various neurotransmitter receptors on bone cells suggested that the nervous system may participate in the control of bone metabolism. However the role of nicotinic acetylcholine receptors (nAChRs) on osteoclastogenesis has not been described in detail.

Materials and methods The authors investigated the presence of nAChR subunits by PCR on osteoclasts (OCs). OCs were defined as tartrate-resistant acid phosphatase (TRAP) positive multinucleated cells, derived from mouse bone marrow, differentiated in the presence of receptor activator of nuclear factor $\mathrm{\kappa B}$ ligand (RANKL) and macrophage colonystimulating factor (M-CSF). Additionally, the authors evaluated the effects of various $\mathrm{nAChR}$ agonists and antagonists on osteoclastogenesis in vitro. OC markers were evaluated by quantitative PCR.

Results PCR investigation confirmed the presence of $\mathrm{nAChR}$ subunits $\alpha 1-9$ and $\beta 1,2$ and 4 in mouse bone marrow derived OCs differentiated in the presence of RANKL and M-CSF. Nicotine dose-dependently and markedly reduced the number 
of OCs by 99\% (inhibitory concentration (IC50): $25 \mu \mathrm{M}$ ). Nicotine also completely reduced the number of pre-OCs, defined as TRAP positive mononuclear cells. Nicotine virtually abrogated osteoclastogenesis, however, the authors observed no difference in total cell number between nicotinetreated and untreated cells; $80 \%$ of cells stained positive for $\mathrm{CD} 11 \mathrm{~b}$ at the termination of the culture suggesting a highly specific effect of nicotine on osteoclastogenesis. Addition of nicotine had no effect on very early stages of osteoclastogenesis, when cells were stimulated only in the presence of M-CSF. Specifically, the effect of nicotine was only apparent in the presence of RANKL indicating interference downstream of RANK. Indeed, when the authors performed quantitative PCR experiments the authors found that mRNA levels of the $M$-CSF receptor c-fms as well as RANK remained unaffected by nicotine, whereas TRAP, cathepsin-K, matrix metalloproteinase 9 as well as nuclear factor of activated $T$ cells, cytoplasmic 1 mRNA levels were markedly reduced by nicotine. The nicotinic agonist epibatidine showed similar effects as nicotine. The non-competitive nicotinic antagonist mecamylamine when given at high doses also inhibited osteoclastogenesis, suggesting that inhibition caused by the agonists might be associated with the desensitisation of the receptor.

Conclusions The authors have shown that nAChR agonists completely block osteoclastogenesis by interfering with $\mathrm{OC}$ precursor differentiation in vitro. The presence of $n A C h R s$ and the effect of $n A C h R$ agonists suggest that nicotine and the cholinergic nervous system may play an important regulatory role in osteoclastogenesis. 